\title{
Method of pectus excavatum measurement based on structured light technique
}

\author{
Wojciech Glinkowski \\ Medical University of Warsaw \\ Department of Orthopaedics and Traumatology of \\ Locomotor System \\ Center of Excellence "TeleOrto" \\ ul. Lindleya 4 \\ 02-005 Warsaw \\ Poland \\ and \\ Medical University of Warsaw \\ Department of Normal and Clinical Anatomy \\ Center of Biostructure \\ ul. Chalubinskiego 5 \\ 02-004 Warsaw \\ Poland \\ and \\ Polish Telemedicine Society \\ Poland
}

\section{Robert Sitnik}

\section{Marcin Witkowski}

Warsaw University of Technology

Institute of Micromechanics and Photonics

ul. Sw. A. Boboli 8

02-525 Warsaw

Poland

\section{Hanna Kocon}

Medical University of Warsaw

Department of Orthopaedics and Traumatology of Locomotor System

Center of Excellence "TeleOrto"

ul. Lindleya 4

02-005 Warsaw

Poland

\section{Pawel Bolewicki}

Warsaw University of Technology

Institute of Micromechanics and Photonics

ul. Sw. A. Boboli 8

02-525 Warsaw

Poland

\section{Andrzej Górecki}

Medical University of Warsaw

Department of Orthopaedics and Traumatology of Locomotor System

Center of Excellence "TeleOrto"

ul. Lindleya 4

02-005 Warsaw

Poland

\section{Introduction}

Pectus excavatum is the most common congenital deformity of the chest wall, with a variable reported incidence from 0.23

Address all correspondence to: Marcin Witkowski, Warsaw University of Technology, Institute of Micromechanics and Photonics, PI. Politechniki 1, Warsaw 00-661 Poland. Tel: +48 22234 8394; Fax: +48 22234 8601; E-mail: m.witkowski@mchtr.pw.edu.pl

\begin{abstract}
We present an automatic method for assessment of pectus excavatum severity based on an optical 3-D markerless shape measurement. A four-directional measurement system based on a structured light projection method is built to capture the shape of the body surface of the patients. The system setup is described and typical measurement parameters are given. The automated data analysis path is explained. Their main steps are: normalization of trunk model orientation, cutting the model into slices, analysis of each slice shape, selecting the proper slice for the assessment of pectus excavatum of the patient, and calculating its shape parameter. We develop a new shape parameter $\left(\mathrm{I}_{3 \mathrm{ds}}\right)$ that shows high correlation with the computed tomography (CT) Haller index widely used for assessment of pectus excavatum. Clinical results and the evaluation of developed indexes are presented. () 2009 Society of Photo-Optical Instrumentation Engineers. [DOI: $10.1117 / 1.3210782]$
\end{abstract}

Keywords: pectus excavatum; thoracic deformity; diagnostics; structured light method; 3-D evaluation.

Paper 09079RR received Mar. 15, 2009; revised manuscript received Jun. 25, 2009; accepted for publication Jun. 30, 2009; published online Aug. 31, 2009. 


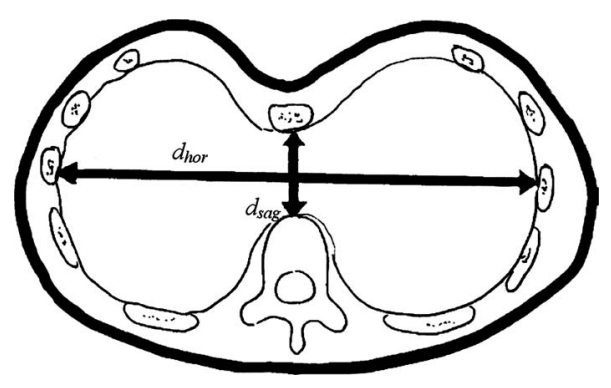

Fig. 1 Dimensions used for Haller index calculation.

and cosmetic indications for surgical treatment gained more importance due to introduction of minimally invasive and cosmetic surgical methods. Physical examination, charts, preoperative photographs, and computed tomography (CT) scans are used to identify pectus excavatum cases. Physical examination should pay attention to asymmetry index, relative length of depression to the sternum, fraction of sternum affected, degree of sternal torsion, and localized versus diffuse morphology. ${ }^{4}$ Preoperative photographs are used to document the deepest point of the pectus, the length of the pectus relative to the sternum, the relative amount of the sternum affected, symmetry of the pectus, localized or diffuse (cup or saucer) morphology, and the presence of flaring of the lower costal margin. Standard photography is not sufficient to document 3-D components of the deformity. However, preoperative photographs can be used to determine chest lines, the area affected by the depression, the vertical length of the deformity, and the length of the sternum affected.

Clinical evaluation and outcome assessment influence the treatment option strongly. Physical examination of patients, measurement of the chest circumference with a metric tape, and anthropometric measurement called chest cyrtometry are performed for basic documentation. ${ }^{5,6}$ The anthropometric index is a simple method to assess the deformity. It is defined as a maximum anteroposterior measurement in the region of greatest deformity divided by measurement of greatest depth of the defect.

Charts, preoperative photographs, and CT scans are usually used to identify pectus excavatum cases. Radiological examination sometimes may additionally define the orientation and relationships of the ribs, costal cartilages, and sternum for preoperative planning. However, plain photographs cannot become concurrent to CT assessment and Haller index (HI) calculation, ${ }^{7,8}$ which remains a gold standard for preoperative planning and decision making. The Haller index calculated from CT scans was developed to provide an objective measure of the depth of the deformity for determining surgical eligibility. ${ }^{9}$ Kim et al. ${ }^{10}$ developed additionally indices that can automatically be extracted by computerized image to describe the depression and asymmetry of the chest wall, and the eccentricity, flatness, and circularity index, to present the deformation of the chest wall in detail. Despite several reported pectus excavatum severity classifications, the Haller index remains the most commonly used. Haller index $H I$ $=d_{\text {hor }} / d_{\text {sag }}$ (Fig. 1) greater than 3.2 is used as one of the criteria for surgical correction. The proposed value was accepted as the cutoff between mild and moderate pectus excavatum.
Single-level HI may not supply sufficient information about chest-wall deformity in all cases. Multiple-level slice examination may supplement an assessment for more accurate diagnosis and preoperative planning. ${ }^{11}$ Due to current trends to reduce overall yearly exposition to ionizing radiation, new methods of pectus excavatum assessment are developed, particularly for repeated monitoring.

\section{Relevant Work}

Although anthropometric index measurement is a very simple method, the human factor may cause a relatively wide range of measurement dispersion. ${ }^{5,6}$

Moiré phototopography ${ }^{12}$ was found in the early 1980 's as the method suitable for 3-D chest assessment. Fortin used a structured light projection system to reconstruct a 3-D surface of a patient's trunk to design a customized spinal brace. ${ }^{13}$ Single directional assessment of back curvature screening has been elaborated in previous studies. ${ }^{14}$ Poncet et al. reported that thoracic outline cross sections can be measured based on 3-D data representing a patient's body surface captured with an optical measurement system. ${ }^{15}$ However, the analysis method presented by them requires an operator's interaction and may imply human factor errors that depend on experience in 3-D measurement data analysis and also clinical knowledge about pectus excavatum assessment.

\section{Aim of the Study}

Promising results of our experiments on 3-D body measurement as well as those described by other researchers caused us to make an attempt to develop an optical 3-D chest measurement system and automatic data processing algorithms to assess the shape and severity of deformity in pectus excavatum cases.

This work presents a high-accuracy optical markerless 3-D measurement system for noninvasive, quick, and relatively inexpensive assessment of pectus excavatum deformity. The system facilitates automatic measurement and data processing software for analysis of the deformity. The process is fully automatic and does not require the operator to interact with the measurement system and data analysis software, which would effect an objective evaluation of the results. To fully automate the whole process, we have developed a new 3-D scan index $\left(\mathrm{I}_{3 \mathrm{ds}}\right)$ that is calculated from a measured chest surface outline. The $\left(\mathrm{I}_{3 \mathrm{ds}}\right)$ index shows correlation with the CT Haller index. Worth stressing is that the measurement method is not based on ionizing radiation, and the measurement process may be repeated frequently without any harm to the patient's health.

\section{Measurement System}

The measurement system built utilizes four modules that measure a patient from four directions (Fig. 2). Directional sensors are optical markerless 3-D measurement systems based on a structured light projection method. ${ }^{16}$ The metrological properties of the system allow for accurate and repeatable measurements, which may be regarded as a good basis for medical examination.

Each module [Fig. 3(a)] consists of a projection unit, which is a digital light processing (DLP) projector (TDP- 


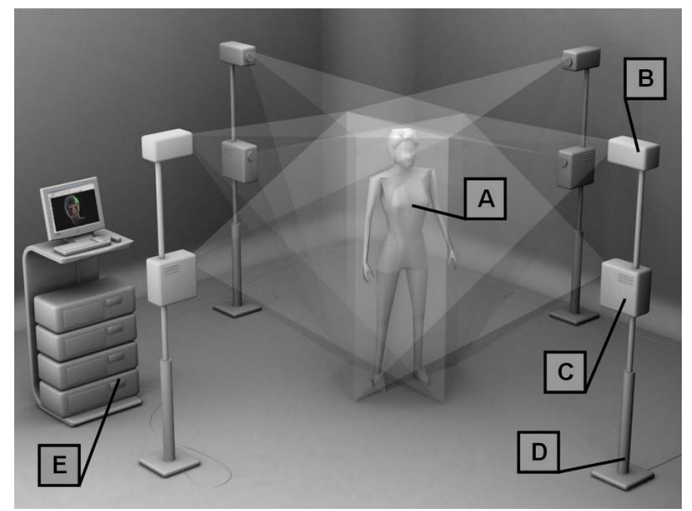

Fig. 2 A visualization of a patient (a) being measured in the system. Each measurement module consists of (b) a CCD camera and (c) a DLP projector (d) fixed to a stand and (e) is controlled by a computer.

MT700 by Toshiba Corporation), and a detection unit, which is a charge coupled device (CCD) camera (Flea B\&W by Point Grey Research Incorporated), During the measurement, certain patterns (sinusoidal fringes and modified binary Grey codes) are projected onto the patient who is standing still inside a calibrated measurement volume.

The shape of the body surface is calculated based on raster deformation [Fig. 3(b)]. To avoid detecting patterns projected by another unit, the adjacent modules are spectrally separated [Fig. 3(c)]. As the directional modules are calibrated in the same global coordinate set, the resulting clouds are merged automatically [Fig. 3(d)].
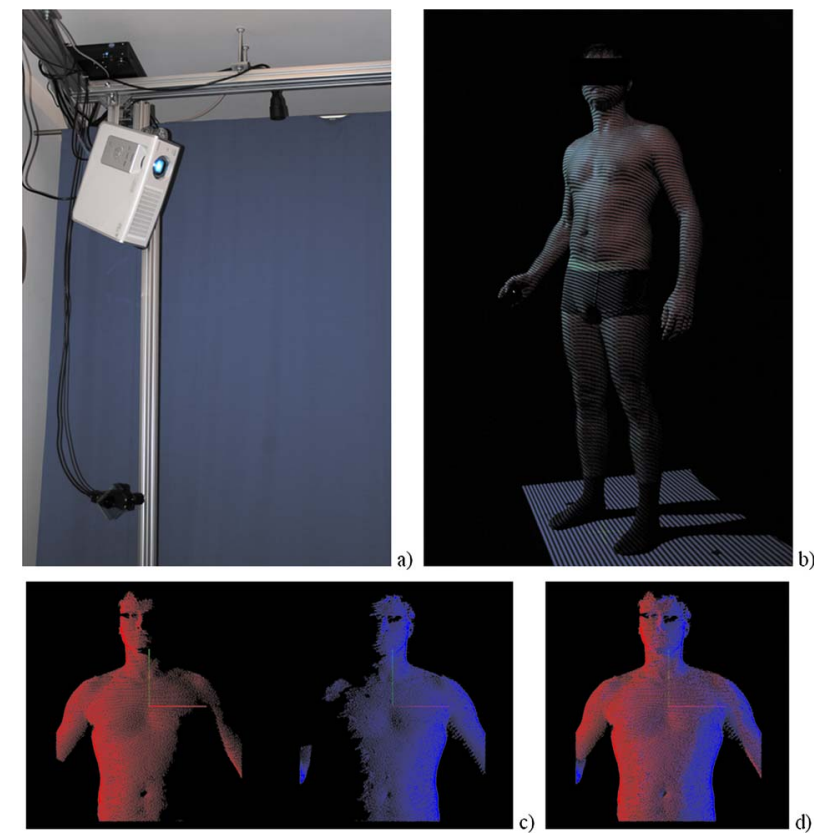

Fig. 3 The measurement setup in the laboratory at the Institute of Micromechanics and Photonics: (a) a directional measurement module fixed to a stand, (b) a healthy individual lighted with a fringe pattern from the module in (a), (c) the two front (+45 and -45 deg) out of four directional point clouds representing the patient's upper body surface, and (d) the front clouds merged automatically based on the global coordinate set calibration.

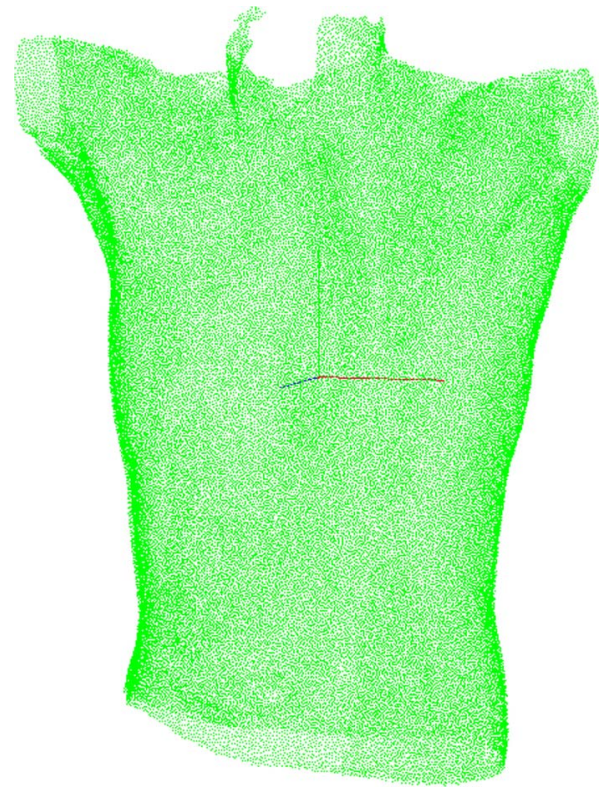

Fig. 4 A 3-D point cloud representing the patient's thoraco-abdominal region measured by the four-directional measurement system.

The dataset produced by the measurement system is in the form of a set of $(x, y, z)$ points that represent the surface of a patient's body. All four directional modules measure in the same global coordinates set. An exemplary result of combined measurement from four directions is shown in Fig. 4. The metrological values of the measurement system are as follows:

- measurement volume size: scalable from $1.0 \times 1.0$ $\times 1.0 \mathrm{~m}$ to $1.5 \times 1.5 \times 2.0 \mathrm{~m}$

- accuracy: 0.2 to $0.4 \mathrm{~mm}$ (depending on the measurement volume size)

- data acquisition time: $0.7 \mathrm{~s}$ (all modules simultaneously)

- maximum number of points: 4 million.

For pectus excavatum assessment, the smaller measurement volume size was used. It reached from 0.7 to $1.7 \mathrm{~m}$ high and its side borders were marked on the floor. During the measurement, the patient was instructed to stand still approximately in the middle of the measurement volume and hold their breath for the measurement time $(1 \mathrm{sec})$. There were no additional requirements regarding the patient's position and orientation related to the measurement system, as the trunk model orientation is initially normalized in the first step of the data processing path.

Stability of the patient during raster projection is essential for the successful measurement, as body movement greater than $1 \mathrm{~mm}$ results in the need for repeating the measurement. Although the projection time is shorter than $1 \mathrm{sec}$, for some patients it may be difficult to remain still. For these patients, additional support, i.e., handrails, may be used.

\section{Automatic Analysis of Chest Surface}

The developed method aims at calculating a 3-D scan index $\left(\mathrm{I}_{3 \mathrm{ds}}\right)$ describing the deformity of the chest in the area where the deformity represents the highest chest depression. The proposed index is calculated based on a transverse slice of the 


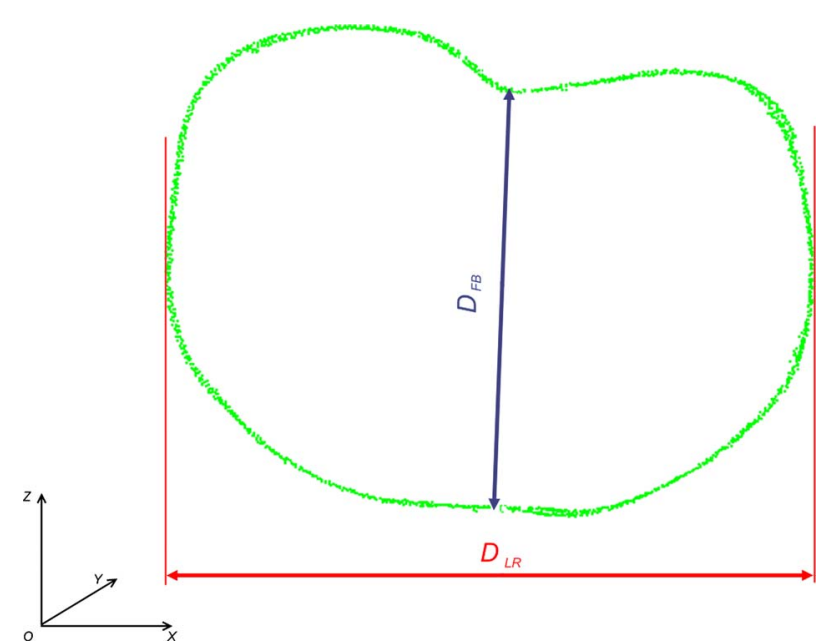

Fig. 5 The slice representative of the patient and the distances used to calculate the $\left(\mathrm{I}_{3 \mathrm{ds}}\right)$ index.

trunk in a way similar to the Haller index to allow for a comparison with the latter. However, it is calculated based on external surfaces. $\left(\mathrm{I}_{3 \mathrm{ds}}\right)$ is defined as the distance from the most depressed portion of the front chest wall to the surface of the back thoracic wall in the midline, divided by the external surface width of the chest at the same level (in the same slice). The $\left(\mathrm{I}_{3 \mathrm{ds}}\right)$ formula is presented below as:

$$
\mathrm{I}_{3 \mathrm{ds}}=\frac{D_{L R}}{D_{F B}},
$$

where $\mathrm{I}_{3 \mathrm{ds}}$ is the calculated index, $D_{L R}$ is the length of the slice along the transversal axis, and $D_{F B}$ is the distance from the most depressed point of the front chest wall to the back chest wall (Fig. 5).

The main steps of the analysis path are as follows. First, the point cloud is divided into preliminary transverse slices. Based on them, the orientation of the torso model is adjusted

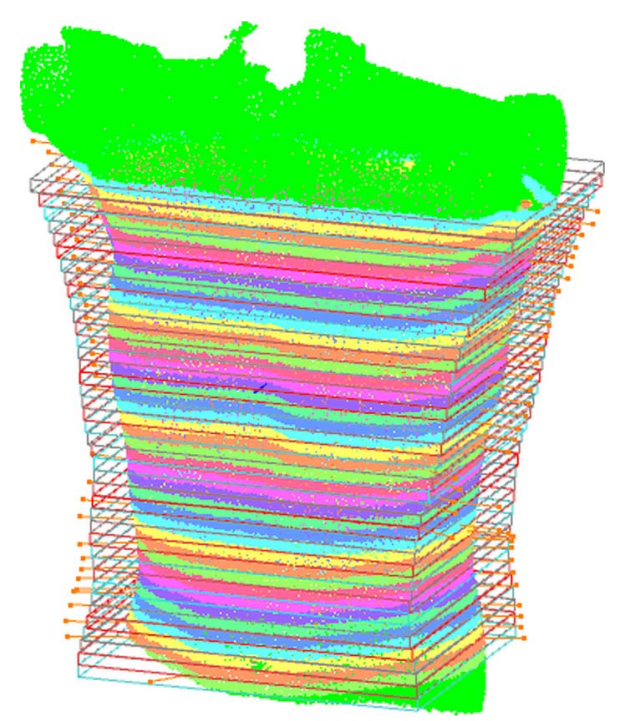

Fig. 6 A torso model sliced with planes horizontal in the coordinate set of the measurement system.

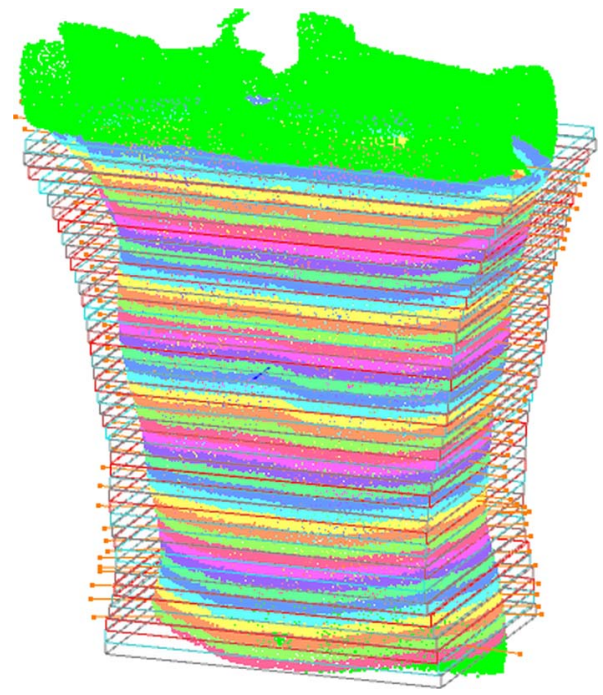

Fig. 7 Transverse slices among which the most deformed slice is searched (open slices have been neglected). Different colors apply to different slices.

to match the normalized coordinate set of the system (see Sec. 5.2). Then the point cloud is sliced again in the proper transverse direction. Next, for all closed slices, values of auxiliary $\left(\mathrm{I}_{3 \mathrm{ds} \text { aux }}\right)$ are calculated and all auxiliary $\left(\mathrm{I}_{3 \mathrm{ds} \text { aux }}\right)$ values are compared. The slice for which $\left(\mathrm{I}_{3 \mathrm{ds} \text { aux }}\right)$ reaches its lowest value for the patient is regarded as the most depressed slice, and its auxiliary index is presented as the $\left(\mathrm{I}_{3 \mathrm{ds}}\right)$ index value representative for the examined patient. The following paragraphs present a more detailed description of the developed algorithm.

\subsection{Preliminary Slices}

The $\left(\mathrm{I}_{3 \mathrm{ds}}\right)$ index is calculated for slices parallel to transverse planes of the body. In consequence, the correct patient orientation in the coordinate system is crucial for further processing. As the point cloud resulting from the measurement may be inaccurately positioned in the measurement volume, an automatic initial adjustment of its orientation is required. To achieve this, the torso model is cut into preliminary slices with planes horizontal in the coordinate set of the measurement system (Fig. 6). The height of the slices and their resulting their number depends on the density of the point cloud. The number of points in one slice has to be big enough for the points to create a solid contour. On the other hand, too-thick slices cause miscalculations of the $\left(\mathrm{I}_{3 \mathrm{ds}}\right)$ index and extension of the processing time. The experiments showed that a $5-\mathrm{mm}$ slice height is optimal for the hardware setup used. The algorithm requires at least ten closed slices to be found.

Slices recognized as open are regarded as incomplete and neglected. Then for each remaining slice its center of mass $(\mathrm{CoM})$ and a long axis are calculated. The long axis is calculated for each slice by minimum root mean square (rms) line fitting for all its points. The resultant line formula defines the position and orientation of this axis. 


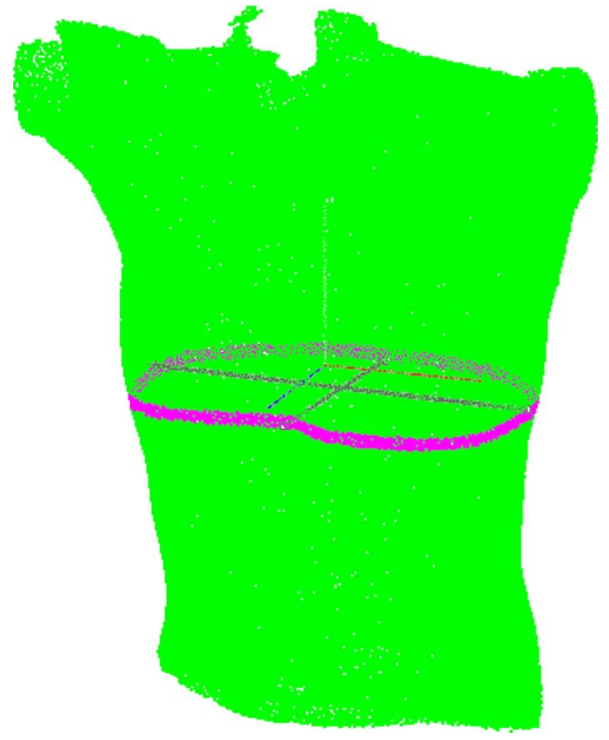

Fig. 8 The slice recognized as the most representative for the examined patient. The characteristic distances used to calculate the $\left(\mathrm{I}_{3 \mathrm{~d}}\right)$ value are also presented.

\subsection{Normalization of Created Model Orientation}

The CoMs of all preliminary slices form a curve that is regarded as an approximation of the model's vertical axis. The orientation of the torso model is adjusted in such a way that the model's vertical axis matches the vertical axis $O Y$ of the coordinate set of the system. Also, the average long axis of the slices is adjusted to match the horizontal $O X$ axis of the system.

\subsection{Accurate Transverse Slices}

When the orientation of the model has been corrected, the point cloud is sliced again with horizontal planes transverse to the patient's torso (Fig. 7). Each slice is checked again as to whether it is a closed or open curve.

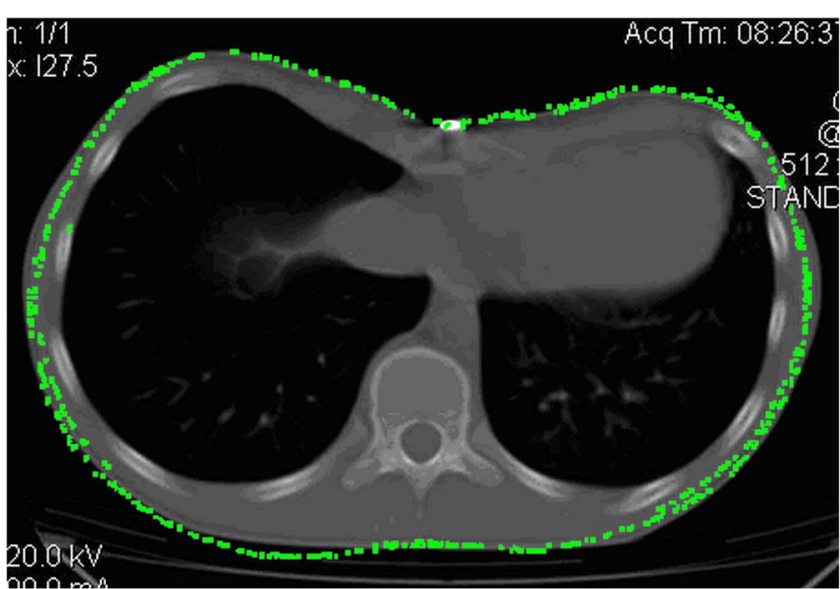

Fig. 9 The CT image and body contour captured with the 3-D optical measurement system (patient 1). (Color online only.)

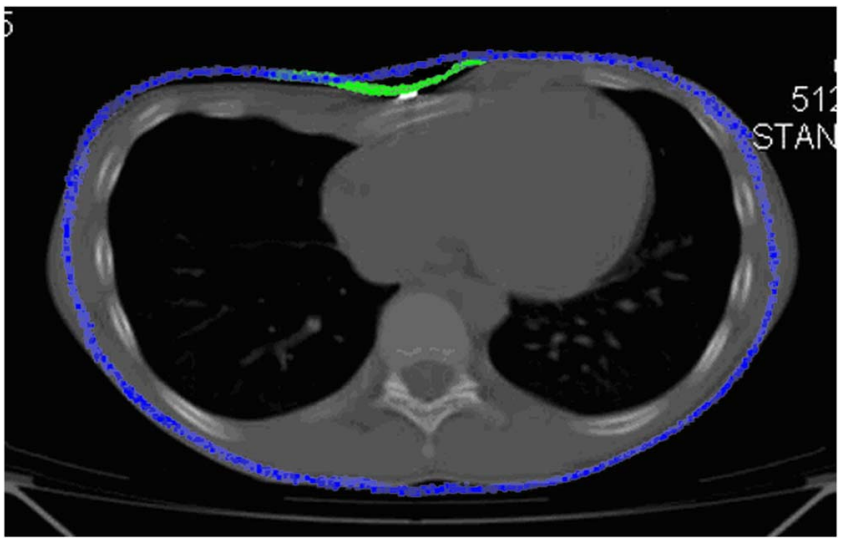

Fig. 10 The CT image and body contour captured with the 3-D optical measurement system (patient 2). The green color represents the contour before the surgical operation, and the blue color represents the contour after the operation. (Color online only.)

\subsection{Calculation of Auxiliary $\left(\mathrm{I}_{3 \mathrm{~d} \text { __aux }}\right)$ Indexes}

The auxiliary $\left(\mathrm{I}_{3 \mathrm{ds} \_a u x}\right)$ index is calculated for each closed slice. The index is a quotient of two distances. The first (placed in the nominator) is the distance from the most depressed point of the front chest wall to the back chest wall. More specifically, it is the shortest distance between two points where normal vectors form an angle exceeding $135 \mathrm{deg}$. The denominator is the width of the slice, which is defined as the size of the slice along the transversal axis.

The slice for which its $\left(\mathrm{I}_{3 \mathrm{ds} \text { _aux }}\right)$ index reaches its minimum is regarded as the most representative slice for the patient

Table 1 Table of measured values of $(\mathrm{CT} \mathrm{HI})$. (CT I $\left.\mathrm{I}_{3 \mathrm{ds}}\right)$, and $\left(\mathrm{I}_{3 \mathrm{ds}}\right)$ indexes for examined patients.

\begin{tabular}{cccc}
\hline Patient & $\mathrm{CT} \mathrm{HI}$ & $\mathrm{CT} \mathrm{I}_{3 \mathrm{ds}}$ & $\mathrm{I}_{3 \mathrm{ds}}$ \\
\hline 1 & 3.67 & 1.83 & 1.75 \\
2 & 3.82 & 1.92 & 1.84 \\
3 & 3.58 & 1.77 & 1.70 \\
4 & 4.05 & 2.02 & 1.92 \\
5 & 3.72 & 1.90 & 1.82 \\
6 & 4.22 & 2.24 & 2.13 \\
7 & 3.77 & 1.97 & 1.87 \\
8 & 3.73 & 1.80 & 1.73 \\
9 & 3.85 & 2.00 & 1.88 \\
10 & 3.86 & 2.00 & 1.91 \\
11 & 3.78 & 1.89 & 1.79 \\
12 & 3.76 & 1.86 & 1.77 \\
Average & 3.82 & 1.93 & 1.84 \\
\hline
\end{tabular}




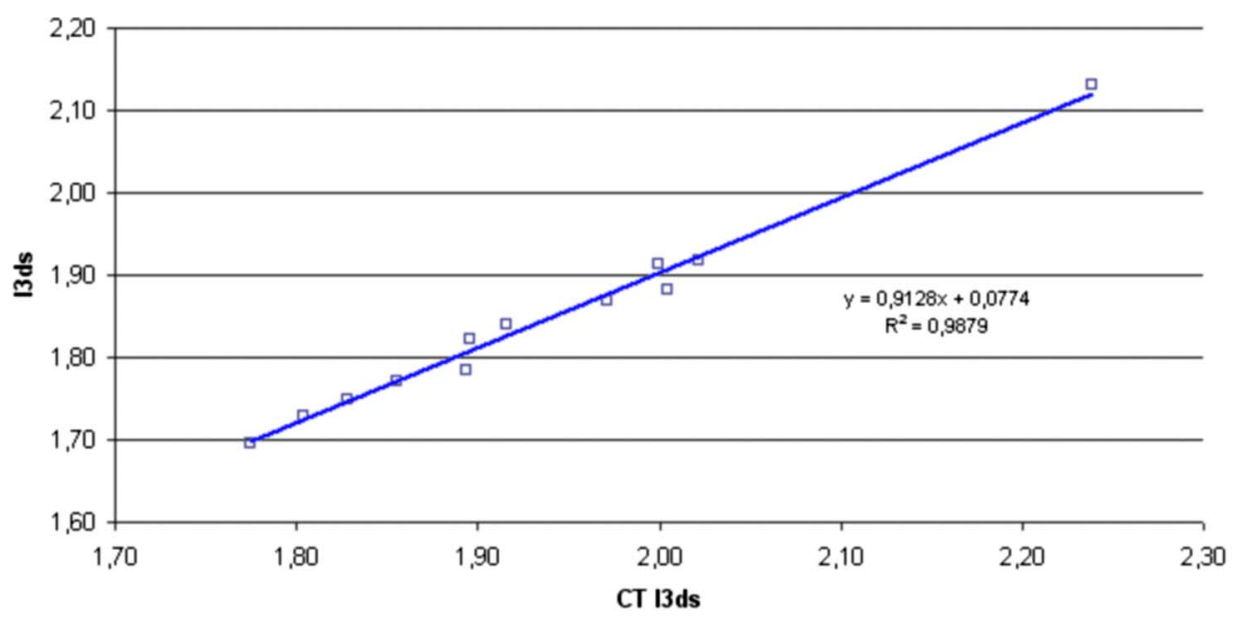

a)

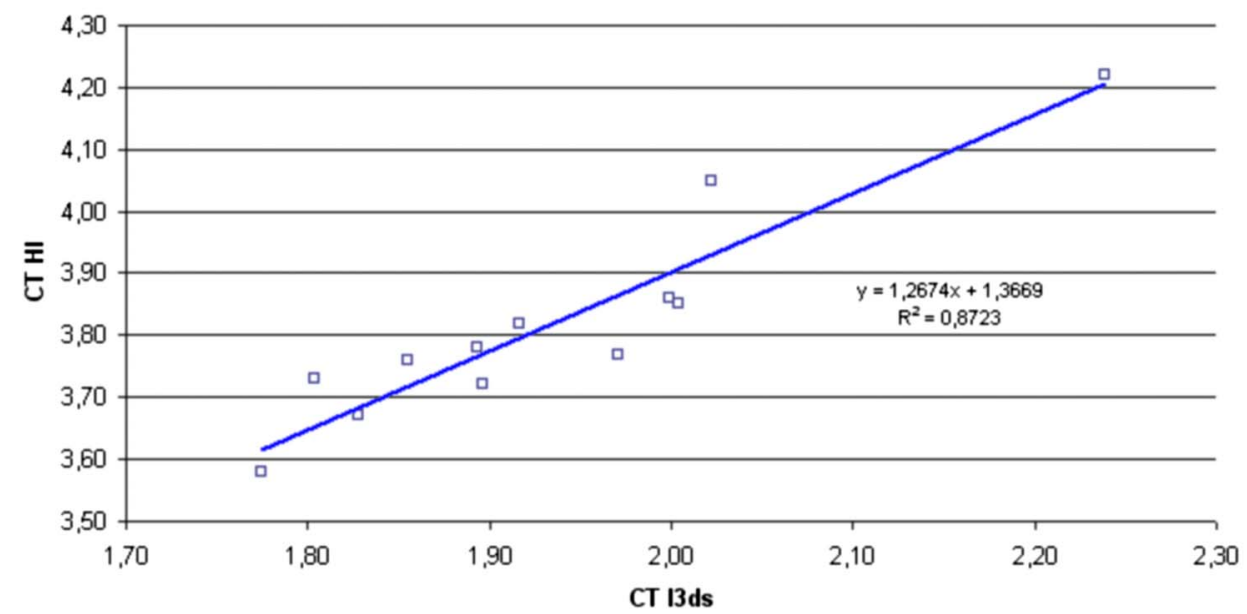

b)

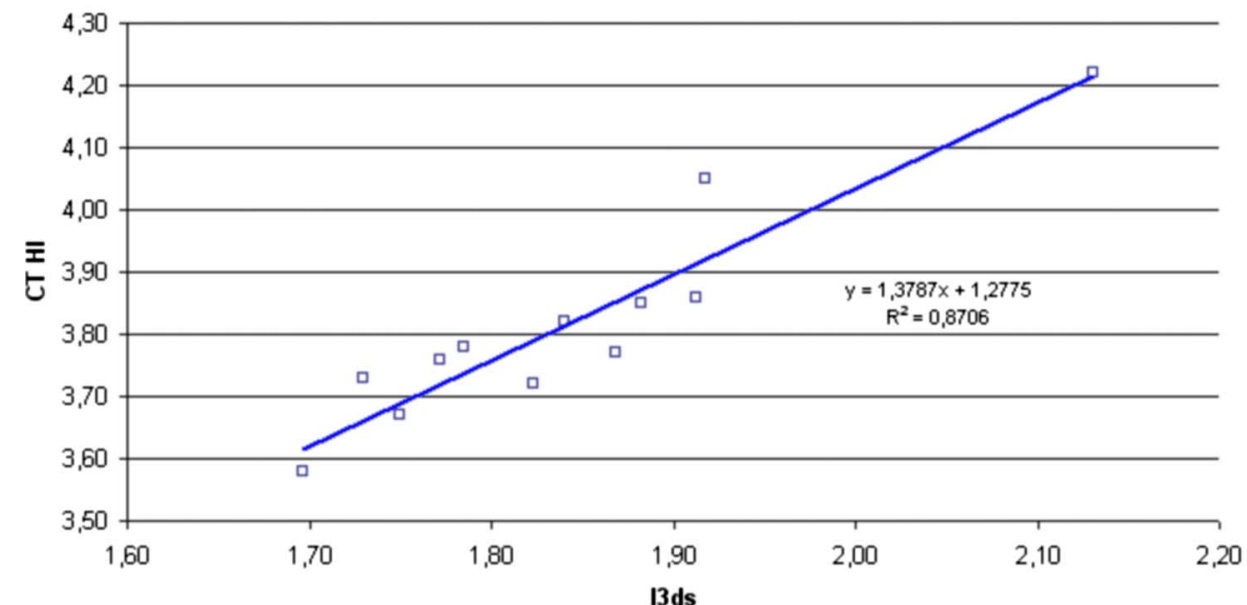

c)

Fig. 11 Graphs presenting Pearson's correlations for tested variables $\left(C \mathrm{I}_{3 \mathrm{ds}}\right)$, $\left(\mathrm{I}_{3 \mathrm{ds}}\right)$, and $(\mathrm{CT} \mathrm{HI}=$ pectus index).

(Fig. 8), and its value is regarded as the $\left(\mathrm{I}_{3 \mathrm{ds}}\right)$ index value representative of the patient.

It is worth repeating that the presented method is fully automatic and does not require any user input. In particular, there are no markers attached to patient's skin and no pointing of the deformation extreme with a mouse is required.

\section{Clinical Examinations}

A prospective study was conducted from November 2007 to December 2008 at a diagnostic laboratory OGX (http:// ogx.mchtr.pw.edu.pl) of the Institute of Micromechanics and Photonics. 12 patients with pectus excavatum $(\mathrm{PE})$ were stud- 
ied. The preliminary series consisted of white adolescent and young adult males an average age of 16 years and 5 months (12 to 19 year). The PE cases were documented through a paired examination of patient 3-D images and CT scans. The CT Haller index was calculated for all cases by an experienced orthopedist. Thorax shapes were measured with the use of the presented measurement system and analyzed according to the presented method.

The CT Haller index ranged from 3.58 to 4.22 (average value 3.82). The following figures present the comparison of slices acquired by means of CT and 3-D optical scanner for patient 1 (Fig. 9) and patient 2 (Fig. 10). The body contour measured with the 3-D scanner is shown in green. There are two contours in Fig 10. The green color represents the contour before the surgery, while the blue color represents the contour after surgery.

There are some differences in the contours of trunk slices imaged with the two modalities. These are caused by different positions of patient bodies during the $\mathrm{CT}$ examination where the patient is supine, and during the 3-D optical scanning procedure where the patient is standing.

Table 1 presents a comparison of values of CT Haller index (CT HI), 3-D scan index calculated based on CT images $\left(\mathrm{CT} \mathrm{I}_{3 \mathrm{ds}}\right)$, and of 3-D scan index calculated based on a 3-D $\operatorname{scan}\left(\mathrm{I}_{3 \mathrm{ds}}\right)$.

\subsection{Correlation between Computed Tomography I $\mathrm{I}_{3 \mathrm{ds}}$ $\mathrm{I}_{3 \mathrm{ds}}$, and Computed Tomography Haller Index $=$ Pectus Index}

Null hypothesis was formulated as a lack of correlation between variables $\left(\mathrm{CT} \mathrm{I}_{3 \mathrm{ds}}\right)$ and $\left(\mathrm{I}_{3 \mathrm{ds}}\right) \mathrm{H}_{0}: \rho=0$. This correlation was tested with Pearson's correlation coefficient at the significance level $\alpha=0.05$ and $\mathrm{R}=0.9939$. A positive correlation between presented variables is present [Fig. 11(a)].

Similarly correlations were tested between $\mathrm{CT}\left(\mathrm{I}_{3 \mathrm{ds}}\right)$ and the Haller index, and also between $\left(\mathrm{I}_{3 \mathrm{ds}}\right)$ and the Haller index. For $\left(\mathrm{CT} \mathrm{I}_{3 \mathrm{ds}}\right)$ and the Haller index, null hypothesis was formulated $\mathrm{H}_{0}: \rho=0$ that no correlation is present.

For preconditioned normal distribution of variables, the hypothesis was tested utilizing Pearson's test of correlation coefficient. The hypothesis was verified at the significance level $\alpha=0.05$ and Pearsons correlation coefficient $\mathrm{R}=0.934$. Due to lower value of $\mathrm{P}$-value $=0.0000$ than the significance level, the null hypothesis was rejected. The positive correlation between variables is seen in Fig. 11(b). $\left(\mathrm{I}_{3 \mathrm{ds}}\right)$ and $\mathrm{HI}$ correlations were tested utilizing Pearson's correlation coefficient. Null hypothesis was set as no correlation was present $\left(\mathrm{H}_{0}: \rho=0\right)$. Considering the normal distribution of variables, the previously mentioned hypothesis was verified at the $\alpha$ $=0.05$ significance level. The calculated Pearson's correlation coefficient was $R=0.9331$. Since $p$-value $(P$-value $=0.0000)$ is lower than the significance level, null hypothesis was rejected. Positive correlation was confirmed between tested variables, as presented in Fig. 11(c). The results of the presented statistical analysis confirm an existing correlation between analyzed variables.

\section{Conclusion}

The study presents a new method for pectus excavatum severity assessment. The deformity analysis is conducted on a 3-D point cloud representing the surface of a patient's torso captured with a four-directional measurement system based on a structured light projection built by us.

A new 3-D scan index $\left(\mathrm{I}_{3 \mathrm{ds}}\right)$ is proposed as a quantitative measure of the deformation. A positive correlation between $\left(\mathrm{I}_{3 \mathrm{ds}}\right)$ and the Haller index is confirmed. The presented method utilizes a fully automatic markerless measurement. Also, the developed data processing path does not need any operator's attention during the analysis process. These two facts make the presented method an objective one.

Contrary to CT scanning, during a measurement based on white light projection, a patient is not exposed to harmful radiation, and there are no contraindications to frequent repetitions of the examination.

\section{Future Work}

In the near future, we will conduct a clinical validation of the presented method on a larger number of patients, and the Haller index based on CT scans will be evaluated by a larger number of experts to avoid subjective assessment errors. Additionally, adaptation of the method to assess other chest wall anomalies, such as pectus carinatum, spinal curvature, and faulty posture in general, is planned.

\section{Acknowledgment}

The project is supported by grant number NR13-0020-04/ 2008 from the Polish Ministry of Science and Higher Education and statutory activity at the Institute of Micromechanics and Photonics, Warsaw University of Technology. The authors would like to express their gratitude to Dorota Kozioł for the statistical analyses used in this work.

\section{References}

1. A. M. Williams and D. C. G. Crabbe, "Pectus deformities of the anterior chest wall," Paed. Resp. Rev. 4, 237-242 (2003).

2. C. S. Chung and N. C. Myrianthopoulos, "Analysis of epidemiologic factors in congenital malformations," Birth Defects Orig Artic Ser. 11(10), 1-22 (1975).

3. S. W. Daunt, J. H. Cohen, and S. F. Miller, "Age-related normal ranges for the Haller index in children," Pediatr. Radiol. 34, 326-330 (2004).

4. M. J. Cartoski, D. Nuss, M. J. Goretsky, V. K. Proudb, D. P. Croitoru, T. Gustin, K. Mitchell, E. Vasser, and R. E. Kelly Jr., "Classification of the dysmorphology of pectus excavatum," J. Pediatr. Surg. 41, 1573-1581 (2006).

5. R. R. Brigato, J. R. Campos, F. B. Jatene, L. F. Moreira, and E. B. Rebeis, "Pectus excavatum: evaluation of Nuss technique by objective methods," Interact. Cardiovasc. Thorac. Surg. 7(6), 1084-1088 (2008).

6. E. B. Rebeis, J. R. Campos, A. Fernandez, L. F. Moreira, and F. B. Jatene, "Anthropometric index for pectus excavatum," Clinics 62(5), 599-606 (2008)

7. J. A. Haller, S. S. Kramer, and S. A. Lietman, "Use of CT scans in selection of patients for pectus excavatum surgery: a preliminary report," J. Pediatr. Surg. 22(10), 904-906 (1987).

8. J. A. Haller, D. W. Shermeta, J. J. Tepas et al., "Correction of pectus excavatum without prostheses or splints: objective measurement of severity and management of asymmetrical deformities," Ann. Thorac. Surg. 26, 73-79 (1978).

9. E. S. Pretorius, J. A. Haller, and E. K. Fishman, "Spiral CT with 3D reconstruction in children requiring reoperation for failure of chest wall growth after pectus excavatum surgery. Preliminary observations," Clin. Imaging 22(2), 108-116 (1998).

10. H. C. Kim, H. J. Park, S. Y. Ham, K. W. Nam, S. Y. Choi, J. S. Oh, H. Choi, G. S. Jeong, S. W. Park, M. G. Kim, and K. Sun, "Development of automatized new indices for radiological assessment of 
chest-wall deformity and its quantitative evaluation," Med. Biol. Eng. Comput. 46, 815-823 (2008).

11. M. L. Lawson, M. Barnes-Eley, B. L. Burke, K. Mitchell, M. E. Katz, Ch. L. Dory, S. F. Miller, D. Nuss, D. P. Croitoru, M. J. Goretsky, and R. E. Kelly Jr., "Reliability of a standardized protocol to calculate cross-sectional chest area and severity indices to evaluate pectus excavatum," J. Pediatr. Surg. 41, 1219-1225 (2006).

12. S. J. Shochat, J. J. Csongradi, G. E. Hartman, and L. A. Rinsky, "Moiré phototopography in the evaluation of anterior chest wall deformities," J. Pediatr. Surg. 16(3), 353-357 (1981).

13. D. Fortin, "A 3-D visualization tool for the design and customization of spinal braces," Comput. Med. Imaging Graph. 31, 614-624 (2007).
14. R. Sitnik, W. Glinkowski, M. Licau, W. Załuski, D. Kozioł, B. Glinkowska, and A. Górecki, "Screening telediagnostics of spinal deformities based on optical 3D shape measurement system and automated data analysis-preliminary report," Proc. 11th Intl. Conf. Med. Inform. Technol., pp. 241-245 (2006).

15. P. Poncet, D. Kravarusic, T. Richart, R. Evison, J. L. Ronsky, A. Alassiri, and D. Sigalet, "Clinical impact of optical imaging with 3-D reconstruction of torso topography in common anterior chest wall anomalies," J. Pediatr. Surg. 42(5), 898-903 (2007).

16. R. Sitnik, M. Kujawińska, and J. Woźnicki, "Digital fringe projection system for large-volume $360 \mathrm{deg}$ shape measurement," Opt. Eng. 41(2), 443-449 (2002). 